\title{
Advanced Abrasive Waterjet for Multimode Machining
}

(Peter) H.-T. Liu, V. Cutler, C. Raghavan, P. Miles,

E. Schubert and N. Webers

Additional information is available at the end of the chapter

\begin{tabular}{|l|l|}
\hline \multicolumn{2}{|c|}{ Author Queries } \\
\hline [AQ1] & Please provide full firstname for the author. \\
\hline [AQ2] & Please check affiliation 2 and amend as necessary. \\
\hline [AQ3] & Please check the term "lightweighting Isogrid pattern" for correctness. \\
\hline$[$ AQ4] & Please provide publisher location for Ref. [1]. \\
\hline
\end{tabular}




\begin{abstract}
Abrasive waterjet (AWJ) possesses inherent technological and manufacturing advantages unmatched by most machine tools. Recent advancements in AWJ processes have enhanced those merits. Multidisciplinary advancements include process automation, position accuracy, cutting models, range of part dimensions, ergonomics, user and environmental friendliness, feature recognition, and others. Among the technological merits, AWJ is material independent and a cold cutting tool, capable of preserving the structural and chemical integrity of parent materials. For heat sensitive materials, AWJ often cuts over 10 times faster than thermal cutting tools such as lasers and electrode discharge machining. Unlike photochemical etching, AWJ is environmentally friendly, producing no toxic byproducts. Additionally, AWJ requires only a single tool assisted with accessories to qualify for multimode machining; it is cost effective with fast turnaround for small and large lots alike. Recent advancements together with relevant R\&D, engineering, and industrial applications will be presented for precision multimode machining from macro to micro scales.
\end{abstract}

Keywords: high pressure pump, micro abrasive waterjet, multimode machining, cold cutting, material independence, heat affected zone, cutting model, multi-passes

\title{
1. Introduction
}

Abrasive waterjet (AWJ) is a machine tool that removes materials by an erosion process of abrasive particles impacting the workpiece at supersonic speeds [1-3]. In [1], the history and fundamentals of waterjet technology and the early stage of the development of micro abrasive waterjet $(\mu \mathrm{AWJ})$ technology is described. This chapter is an update to report the 
progresses in the evolution of $\mu \mathrm{AWJ}$ and its impact on the overall advancement of waterjet technology.

AWJ inherently possesses several technological and manufacturing merits unmatched by most other tools [1]. The ones that are most relevant for precision machining are revisited below and expanded throughout this article.

- Material independence - cuts virtually any material, thin and thick

- Cold cutting - induces no heat affected zone (HAZ) and preserves structural and chemical integrity of parent materials

- Low force exerted on workpiece

- One tool qualified for multimode machining

- Broad range of part size from macro to micro scales

- No tooling requirement - cost effective with fast turnaround

In a 2005 marketing report, Frost and Sullivan stated that waterjet machine tools emerged as the fastest growing segment of the overall machine tool industry in the last decade, and this trend is expected to continue. ${ }^{1}$ The lack of awareness among potential end-users, however, posed a stiff challenge to market participants on increasing the end-user base. Since then, waterjet technology has made advancements to take full advantage of its inherent merits. Waterjet performance has been elevated to the degree that it competes on an equal footing with conventional tools such as lasers, electronic discharge machining (EDM), and photochemical etching. In some cases, its performance greatly exceeds those of its conventional counterparts. The lack of awareness of these merits, though, still presents a considerable challenge to a broader acceptance as a precision machine tool.

\section{Technical approach}

The evolution of waterjet technology has focused on the development of software, hardware, and machining processes to take advantage of technological and manufacturing benefits. These developments focused on automating machining processes, improving machining precision and efficiency, minimizing environmental impact, enhancing ergonomics, ensuring user friendliness, and broadening capabilities toward multimode machining. At OMAX Corporation, this included software development of the Intelli-MAX Software Suite to upgrade to new generations of cutting models and add new machining features aimed at precision and automated machining; hardware development and commercialization of micro abrasive waterjet $(\mu \mathrm{AWJ})$ for meso-micro machining and the development of novel processes for machining various features; and process development of novel concepts for machining various features.

$\overline{{ }^{1} F r o s t ~ a n d ~ S u l l i v a n ~-~ " T h e ~ W o r l d ~ W a t e r j e t ~ C u t t i n g ~ T o o l s ~ M a r k e t s " ~ D a t e ~ P u b l i s h e d: ~} 30$ Aug 2005 (www.frost.com) 


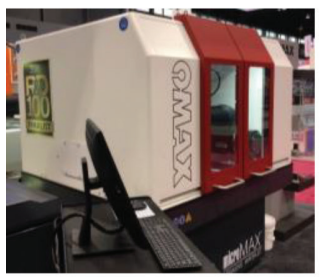

a.

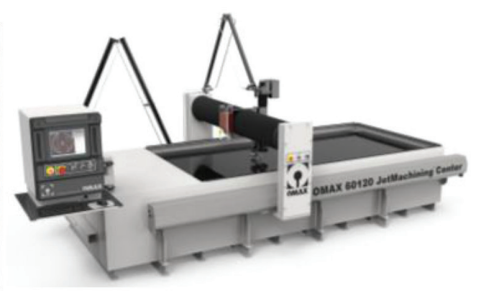

b.

Figure 1. (a) MicroMAX and (b) 60120 JMC. 


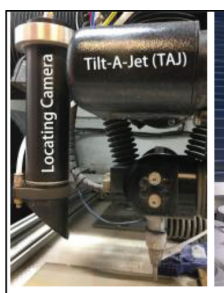

a.

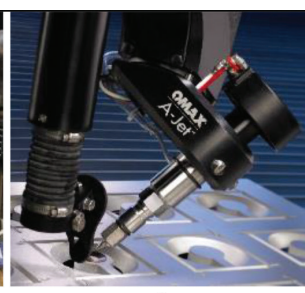

b.

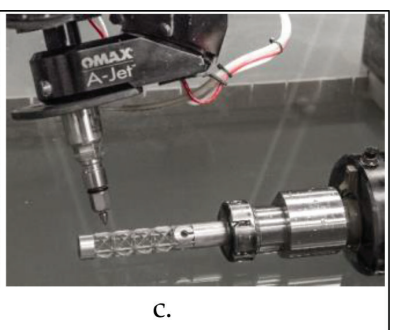

Figure 2. Accessories for 3D machining (a) TAJ (b) A-Jet (c) Rotary Axis.

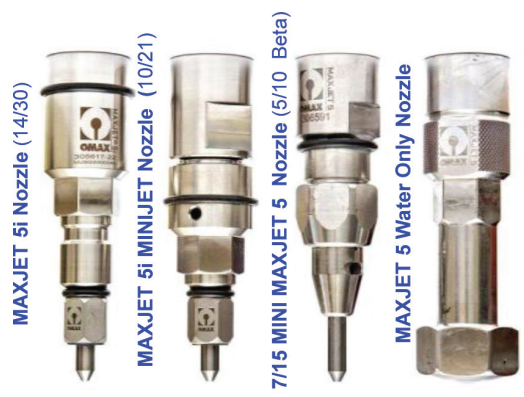

Figure 3. Waterjet nozzles.

Intelligent software - The JMCs are controlled by a suite of software programs built around the patented motion control to automate the AWJ machining processes. Samples of the software suite are listed below.

\subsubsection{Operational software}

- LAYOUT is a full-featured CAD program created and designed to work with JMCs. Part drawings can be created by using a full set of drawing tools, importing a drawing from another CAD program in standard format such as DXF, or tracing a drawing or photograph. The toolpath of that part can then be created with LAYOUT.

- MAKE actually controls the JMC to create parts with several simple steps: (1) open a toolpath file created by LAYOUT (or another CAD/CAM drawing tool), (2) choose the material you want to use and its thickness (from which the exact nozzle motions required to make the part are calculated accurately to within 5/1000ths of an inch, and (3) click on the "Begin Machining" to begin machining parts. 
- Intelli-MAX ${ }^{\circledR}$ - a suite of new technologies integrated into the OMAX JMCs to enhance the performance of AWJ machining. It is designed to make higher tolerance parts faster - faster and with higher tolerance than any other AWJ systems. The suite has several software modules including Intelli-NEST for part nesting, Intelli-PIERCE for hole piercing, IntelliTAPER to minimize edge taper, and Intelli-CORNER to corner compensation.

\section{Results}

One of the most recent advancements in waterjet technology was the development of micro abrasive waterjet ( $\mu \mathrm{AWJ}$ ) technology for meso-micro machining. The merits of cold cutting, material independence, and low side force exertion on workpieces are keys to elevate the $\mu \mathrm{AWJ}$ as a precision meso-micro machine tool.

For cutting heat-sensitive materials, waterjet is superior to thermally based machine tools such as lasers, electric discharge machining (EDM), plasma cutting, and others. The heat generated by these tools induces a heat-affected zone (HAZ) that alters the structural and chemical properties of the parent material. For thin materials, for example, the heat damage by $\mathrm{CO}_{2}$ lasers results in considerable part warpage, formation of slag, or even vaporization of materials $[2,4]$. The HAZ must be removed or minimized. Removal often requires grinding that is time consuming whereas minimization of the HAZ requires significant reduction in cutting power and therefore cutting speed.

Many machine tools are material limited. For example, lasers have difficulty cutting reflective materials such as copper; EDM cannot cut nonconductive materials; CNC hard tools meet with considerable challenges to cut hardened metals with large Rockwell indices. On the other hand, AWJ cuts most of these materials for a wide range of part size and thickness from macro to micro scales. In fact, AWJ cuts titanium $34 \%$ faster than stainless steel.

The low side force exertion on workpieces enables the AWJ to machine thin separations between features. Although the diameter of the $\mu \mathrm{AWJ}$ nozzle is only capable of machining features such as the kerf width of slots and the diameter of holes in the meso scale range $(>200 \mu \mathrm{m})$, the separation or wall between these features is approaching the micro scale range $(<100 \mu \mathrm{m})$ [5]. Such a meso-micro machining capability is unmatched by most machine tools that do not offer the combination of cold cutting and low side force exertion.

By adding the MicroMAX into its product line, OMAX has established the full capability of multimode machining of most materials from macro to micro scales - the " $7 \mathrm{M}$ " advantage [3]. Considerable efforts have been devoted to conducting cutting tests and presenting the samples to demonstrate the versatility of waterjet technology as a whole. Selected tests and samples are presented herein.

\subsection{High pressure pump}

The only method we have to produce these very high pressures is through reciprocating motion. There is no turbine or other "continuous" mechanism that can do this. The two 
types of electrical prime movers are the electric motor that rotates and a coil or solenoid that can directly produce reciprocating motion. The other prime mover is an internal combustion engine that starts out producing exactly the kind of reciprocating motion we require but in most cases this is converted to rotary motion through a crankshaft. This is then converted back to reciprocating motion in the pump. Waterjet pumps for industrial use are run by electric motors. Pumps for field use tend to be powered by internal combustion engines.

1. Electric motor-intensifier: These are the earliest systems, with the first commercially viable system having been developed by McCartney MFG originally for pumping catalyst in the polyethelene industry. ${ }^{2}$ The electric motor drives a hydraulic pump. This hydraulic pressure is routed through a four-way valve system to either side of a hydraulic intensifier that results in reciprocating action and high pressure.

2. Electric motor-direct drive pump: This approach eliminates the hydraulic circuit. An electric motor drives a crankshaft that converts rotary motion to reciprocating motion. These systems can also be run by an internal combustion engine for field applications.

3. Low speed electric servo motor - intensifier: This uses a ball screw to convert low speed rotary motion to low speed reciprocating motion.

Hydraulic horsepower: This is the HP delivered at the nozzle. All the power consumed by the electric motor ends up either as hydraulic HP that is the useful power, or as wasted power in the form of heat.

Efficiency: The electric power delivered to the motor is used up in the following ways:

\section{- Resistance heating}

- Losses in the electric motor windings are proportional to the square of the current $\left(\mathrm{i}^{\wedge} 2 \mathrm{R}\right)$. Motors can be designer with various efficiencies depending on windings. A normal efficiency of an electric motor is in the $90 \%$ range.

\section{- Conversion of rotary to linear motion}

- The crankshaft is the most efficient method of doing this, as the forces are transmitted between two cylindrical surfaces with a lubrication film between them. The crankcase oil in a direct drive pump should not generally require any cooling system.

- The hydraulic intensifier is the least efficient as it first converts the rotary motion of the motor to reciprocating motion of the hydraulic pump plungers which then pump a flow rate of hydraulic fluid 20-33 times the cutting water flow rate through a loop. This consists of passages in four-way valves and relief valves, causing pressure drops and heating. This fluid then has to move a large diameter piston that is connected to a smaller diameter plunger, and then return to the holding tank from where it is recirculated. The heat accumulates in the oil and has to be removed by pumping cold water through a heat 
exchanger or a chiller. The cooling water flow rate may be 4-6 times the water used for cutting.

- In the low speed servo motor system, a servo motor drives a ball screw to convert rotary to linear motion. The ball screw is ideal for accurate position control of the XYZ axes but is highly inefficient at converting large amounts of power and huge forces from rotary to linear motion. These forces have to be conveyed across the small surface areas of the balls in the ball screw, creating a lubrication challenge. The lubrication system of the ball screw has to be separately cooled.

- Friction between the plungers and the guide bushings and dynamic seals create a small amount of heat in all pumps.

- Check Valves create heat when they leak and this is taken away by the cutting water that can also be used to cool the plungers and the dynamic seals.

Useful power/wasted power: This is the ratio of the two powers referred to above - the good vs. the bad. The lower the ratio, the worse the pump. The ratio for an intensifier can be one third that of an efficient direct drive pump.

Check valve design: A good seal requires high, even contact stress in the sealing zone. A ball on a cone does precisely this along a circle. A flat poppet on a flat seat is not the ideal way to seal a high pressure system. The probability of random debris getting between two flat surfaces is vastly higher than the probability of debris getting precisely on the ball-seat circle of contact. Second, if debris gets in between the flat surfaces it has no chance of escaping, whereas it gets pushed to one side or the other by the spherical surface of the ball and not cause damage. Third, the metal surfaces of the flat poppet and seat get eroded easily by high pressure water sneaking past on almost every stroke as the two surfaces cannot close in a manner precisely parallel to each other. These flat surfaces need frequent lapping, leading to more maintenance.

Constant and variable speed control: The bore of an orifice may vary by $2.5 \%$. At a certain pressure, the difference in flow rate between these extreme sizes will be $5 \%$. If a pump is set up to run at constant speed, producing a constant flow rate, the pressure drop across this range of orifice sizes will vary by $10 \%$. In order to operate at a set pressure, a constant speed pump will have to be run at a higher speed to accommodate the larger size orifice and most of the time it will be dumping the extra water. Also, as the seals wear and the check valves erode, the output flow will drop and the pump will have to compensate for this and run constantly at an even higher speed. Constant speed pumps therefore run at about $10-13 \%$ higher speed than variable speed pumps and all this extra output is wasted. The variable frequency drive (VFD) adjusts the speed for the required pressure and avoids wastage.

When piercing holes, it is advantageous to drop the pressure to a piercing pressure. Doing this is easy with the VFD. An important application for waterjets is cutting composites and brittle piercing. Drilling starter holes in composites and in brittle materials requires the pump to shut off and start with the nozzle open. Direct Drive Pumps with a VFD can do this easily. 


\subsection{Micro AWJ technology}

Under the support of an NSF SBIR Phase II grant, OMAX developed and commercialized $\mu$ AWJ technology, culminating the MicroMAX JetMachining Center for precision meso-micro machining. ${ }^{3}$ The MicroMAX was subsequently upgraded by incorporating the TAJ for taper compensation and the Rotary Axis for machining axisymmetric features on tubes and cylindrical workpieces. The MicroMAX was named a Finalist of the 2016 R\&D 100 Award. The technological innovation and success in commercialization of the MicroMAX has led to OMAX's reception of the U.S. Small Business Administration (SBA) 2016 Tibbetts Award. NSF subsequently selected OMAX as a success story for its SBIR/STTR program (https://www.sbir.gov/node/1308555).

The MicroMAX takes advantage of most of the merits of waterjet technology. Success in making the MicroMAX available commercially has greatly broadened the waterjet machining applications. The meso-micro machining capability has led to penetrating several industrial sectors in which conventional waterjets are inadequate for $R \& D$, prototyping, and production applications. These sectors include but are not limited to aerospace, biomedical, electronic/ optic, engineering, and military applications.

For precision AWJ machining, consistent abrasive flow rate is essential. Garnet is mostly used for AWJ machining because of its low cost and superior performance as the abrasive. A rule of thumb to prevent nozzle clogging with abrasives is to use abrasives with mean particle size no larger than $1 / 3$ of the bore diameter of the mixing tube. This is to avoid bridging of two large particles inside the bore. With the downsizing of AWJ nozzles, the particle size of the abrasive is proportionally reduced accordingly. It is well known that the finer the particle, the more difficult for it to flow under gravity feed. One of the common problems of feeding fine abrasive from a hopper is the formation of rat holes, resulting in unsteady mass flow [6]. As the rat holes are formed, flushing or flooding of fine abrasives would result when a positive pressure gradient builds up locally near the nozzle. Packing of fine abrasives also leads to positive pressure buildup. Under certain circumstances, a negative pressure gradient could build up just upstream of the nozzle. The presence of negative pressure gradient would reduce the flow rate of fine abrasives through the nozzle. In other words, fine abrasives flowing through the hopper would experience unsteady flow rate under the influence of buildups of positive and/or negative pressure gradients inside the hopper. The abrasive ceases to flow when the rate holes are fully developed. For the $5 / 10$ nozzle, the finest abrasives can be used to assure consistent feeding is 240 mesh with a mean particle size of $60 \mu \mathrm{m}$. Since the surface roughness of AWJ-machined edges is proportional to the particle size, finer garnet such as 320 mesh with a mean particle size of $30 \mu \mathrm{m}$ is often used to reduce surface roughness. Novel processes were developed to improve the flowability of fine abrasive 320 mesh and finer (US Patent 8920213 B2). Figure 4a and b shows three photographs of the flow patterns of unprocessed and processed garnet, respectively. When examining flow patterns of 320-mesh garnet exiting the feed gate of the hopper, the unsteadiness and inconsistency of the flow patterns of the unprocessed garnet is evident. Cutting with unprocessed fine garnet would lead to wavy kerf width and even skipped cutting [7].

Figure 5 shows a displaly board highlighting $\mu \mathrm{AWJ}$ machined 2D and 3D parts cut from various materials such as metals (aluminum, steel, and titanium), nonmetal (glass, ceramics, carbon

3There are five US patents and one PCT patent application pending for $\mu \mathrm{AWJ}$ technology 


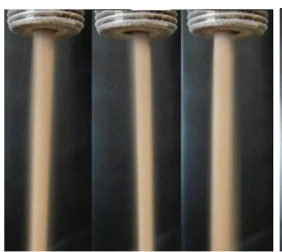

a.

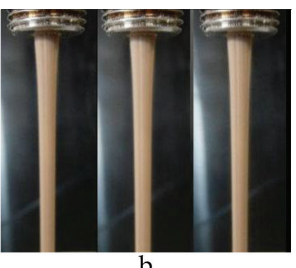

b.

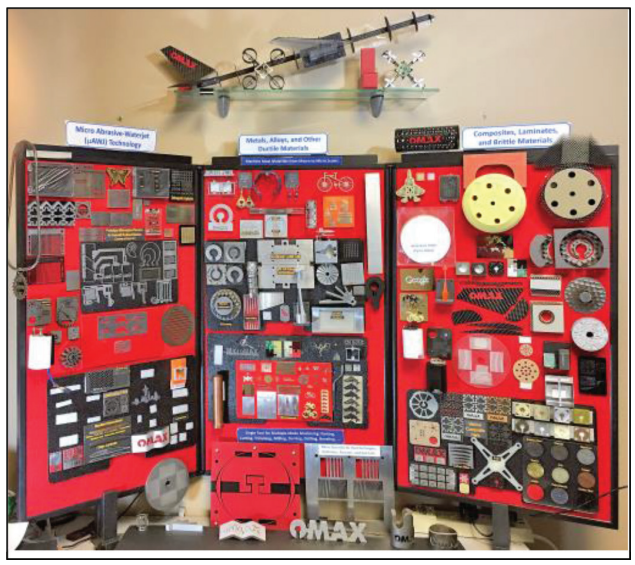

Figure 5. Photographs of $\mu \mathrm{AWJ}$-machined parts-An overview.

fiber, acrylic, polycarbonate, Garolite (G10), Poly-Ether-Ether-Ketone (PEEK), and honeycomb. Also, a simulated nanomaterial with large gradients of nonlinear material properties has been cut with AWJ [7]. These displays clearly demonstrate the merits of abrasive waterjet technology for material independence, no tooling requirements, and one single tool for multimode machining. There is simply no other machine tool capable of machining such a wide range of materials.

As a cold cutting tool that is materials independent, the $\mu \mathrm{AWJ}$ was demonstrated to machine large-aspect-ratio slots on a $2.2 \mathrm{~mm}$ thick $440 \mathrm{C}$ stainless steel sheet that was heat treated to a Rockwell index of $R_{c}=58$. The part is a bonding extender for lapping thin-film ceramic substrates. This $\mu$ AWJ machined part was cut on the MicroMAX using the 5/10 nozzle with 240-mesh garnet. Figure 6 illustrates the $\mu$ AWJ machined part using the 5/10 nozzle with the 240 -mesh garnet. Figure $\mathbf{6 a}$ and $\mathbf{b}$ correspond to the photographs of the entry and exit surface of the part. Pockets and patterns were precut on the blank before waterjet machining. The slots consisted of widths as narrow as $0.3 \mathrm{~mm}$ and lengths as long as $260 \mathrm{~mm}$. In the absence of the $\mathrm{HAZ}$, it took a single pass of the waterjet to machine the part in 23 minutes. 


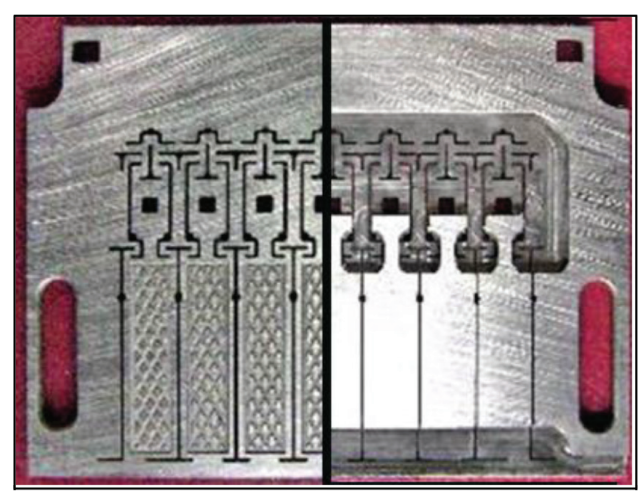

a.

b.

Figure 6. $\mu \mathrm{AWJ}$-machined complex slot patterns on hardened steel (Courtesy of Competitive Engineering) [2] (a) Entry side (b) Exit side.

For such narrow slots with large aspect ratios machined on highly hardened steel, it is extremely difficult if not impossible to cut using $\mathrm{CNC}$ hard tools as they often do not have the stiffness and tend to wear too rapidly to achieve the required tolerance. The current method to machine the part is by wire EDM. The EDM process requires three passes to cut each slot in order to minimize the HAZ. As a result, it took over 6 hours to cut the part. In other words, the cutting speed of the waterjet is better than 15 times faster than the wire EDM for comparable cutting quality.

With the TAJ activated, nearly taperless or square edges can be readily machined with waterjets. Several precision devices critically rely on square edges to achieve their optimum performance. Mechanical flexures are often used for accurate force measurements, precision motion control, and mitigation of backlash. In collaborating with MIT Mechanical Engineering, OMAX used the MicroMAX to machine prototypes of nonlinear load cells with large-aspectratio of thin flexures [8]. The patented design was capable of five orders of force range and its superior performance was verified through laboratory experiments $[9,10]$. The close agreement between the theory and the experimental results was attributed to the nearly taperless edges of the large-aspect-ratio flexures.

As a part of Asteroid Redirection Mission (ARM) program, the Jet Propulsion Laboratory (JPL) of NASA has been developing prototypes of flexure-based microsplines to serve as the asteroid gripping device. The flexures consisting of several spring-like elements were originally machined with the wire EDM that must be cut with multiple passes at low speeds to minimize the heat damage in the presence of the HAZ such as surface hardening on the cut edges and distortion of the spring-like flexure elements. In collaborating with JPL, OMAX conducted a series of tests to machine several 3.2-mm-thick aluminum flexures. The single-pass cutting tests were conducted on the MicroMAX with the 7/15 nozzle together with 240-mesh 
garnet. Figure 7 illustrates several flexure elements that were supported only at two ends. With the TAJ activated, the cold cutting with extremely low side force exertion is essential for cutting such flexure elements with nearly taperless edges and very little distortion. The performance of the MicroMAX also met NASA's precision requirements. Based on the times required to machine these parts, the cost ratio of the waterjet and wire EDM was 1:14, leading to a cost saving of $93 \%$. JPL has adopted the MicroMAX as one of the primary tool to continue the development and refinement of microsplines for the asteroid gripping device.

The Rotary Axis facilitates machining of axisymmetric features on tubes and round bars. Initially the LAYOUT drawing is the same as that of the 2D part. The features along the Y-axis are then converted to those in the rotary axis via the X-data, an algorithm that lets one input "extra" data for any entity in a drawing. After the tool path of the drawing is created, MAKE cuts the part by controlling the motion of the Rotary Axis to machine the axisymmetric features on the part. Figure 8a illustrates an interlocking link structure in a tube machined with AWJ.Machining theinterlocking feature would bechallenging for other machine tools. Figure $8 \mathbf{b}$ illustrates a titanium mesh cage, an implant used in spinal surgery to replace and reinforce the anterior column. A sacrificial rod was inserted into the tube while machining to protect the opposite wall from damaged by the spent AWJ. a titanium mesh cage, an implant used in spinal surgery to replace and reinforce the anterior column. A sacrificial rod was inserted into the tube while machining to protect the opposite wall from damaged by the spent AWJ.

\subsection{Versatility of AWJ technology}

With four product lines of waterjet systems equipped with accessories for 2D/3D machining and nozzles for wide range of part size and thickness, OMAX has established the full capability for multimode machining of most materials from macro to micro scales - the "7 M" advantage [3]. Several publications have been devoted to demonstrating the versatility of waterjet in terms of material independence and precision meso-micro machining capability [2-5, 7-10]. Inside the Engineering and the Demonstration Laboratories, cutting tests continue taking place to look for new applications on new materials. A part of the tests was conducted

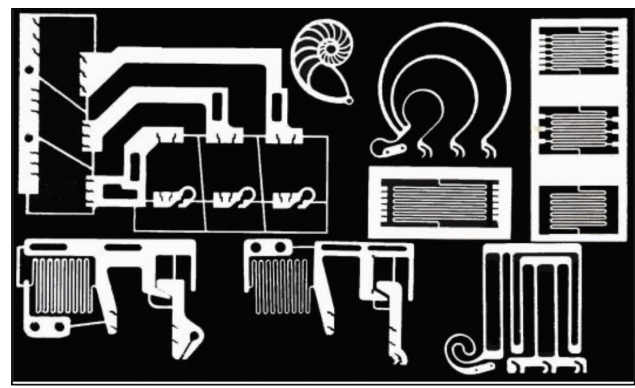

Figure 7. $\mu \mathrm{AWJ}$-machined aluminum flexures with flimsy spring-like elements (courtesy of NASA/JPL). 


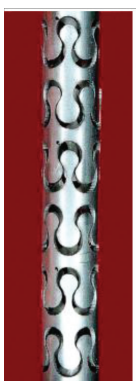

a.

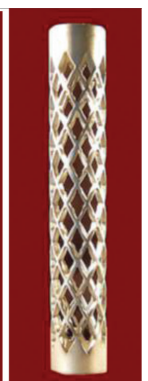

b.

Figure 8. Two cylindrical parts machined with the rotary axis.

by the in-house R\&D and Engineering Group. Many of them were requested from prospective clients before committing to purchase one or more of the machines. In this subsection, several applications to demonstrate the versatility of waterjet technology are described, in particular, those applications that are unique to waterjet technology.

\subsubsection{D machining}

The spent AWJ still consists considerable erosion power, if "not tamed," could cause damage either to the operator or workpiece around the cutting nozzle. In other words, AWJs are not inherently suitable for 3D machining, particularly for parts with complex 3D features. Because the simplest and most effective means to dissipate the residual energy of spent abrasives is to let the spent AWJ shoot into a column of still water, most AWJ systems are built on top of a water tank that also serves to support the traversing mechanism. Such AWJ systems are generally designed for 2D machining. Novel methods and accessories were developed, within the constraints of operational safety, to machine 3D parts using 2D AWJ systems [11].

One of the simple methods to machine a $3 \mathrm{D}$ part on a $2 \mathrm{D}$ platform is to machine it multiple times in different orientations. As an example, Figure 9 illustrates a model fighter plane machined on an aluminum rectangular block in three orientations.

Another example was to build a 3D assembly using many 2D components. Figure 10 illustrates a model Boeing 777 aircraft (right half) that was assembled from AWJ-machined wing and nacelle cross sections, stabilizer, and rudders made from thin sheets of carbon fiber. Selected wing cross sections are shown in the upper left corner.

The Intelli-MAX Software Suite has incorporated several programs for machining parametric shapes, or pre-configured shapes that use equations to machine a shape without having to create the tool path first. One such program is the internal and external Gear, Rack and Sprocket Generator in both U.S. and metric standards. Using the 5/10 nozzle on the MicroMAX, several sets of miniature planetary gears made from titanium, PEEK (with and without fiber reinforce- 


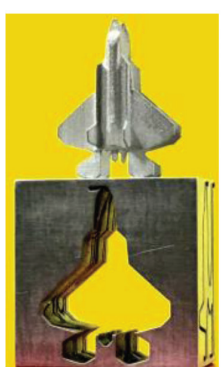

a.

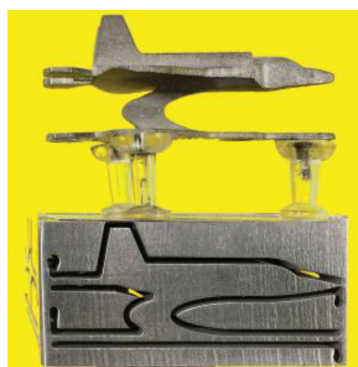

b.

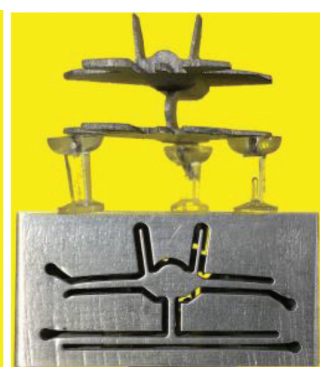

C.

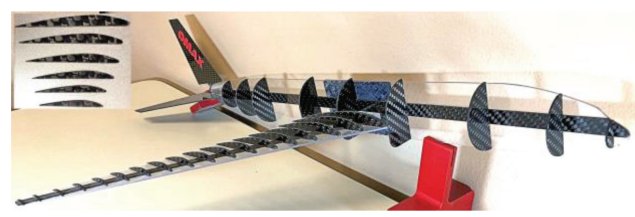

Figure 10. A Boeing 777 aircraft model assembled from AWJ-cut components made from carbon fiber [3].

ment) were machined and assembled into operating models [3]. One of the common gears is the cycloidal gear that is designed for watch making. Figure 11 illustrates a set of miniature cycloidal gears cut with the 5/10 nozzle on the MicroMAX. The gears were made from titanium sheet $2.0 \mathrm{~mm}$ thick. They were assembled into two decks of gears driven by a micro motor (a 298:1 $71 \mathrm{rpm}$ micro spur gear head motor manufactured by Solarobotics, Model GM14a). The lower deck consists of a large gear (19.3 mm OD) and two small gears (5.4 mm OD). The upper deck consists of a large gear (12.7 mm OD) and three small gears (3.61 mm OD). The two decks of gears were separated by an acrylic plate. The two large gears were mounted on a common shaft that is driven by the micro motor powered by a $3 \mathrm{~V}$ button battery (Panasonic CR2477). The assembled AWJ as-cut gears run quite smoothly, demonstrating the adequacy of the precision of the MicroMAX. Our goal is to machine the components of a pocket watch and assemble the watch as a means to demonstrate the capability of the MicroMAX for precision micromachining.

As the first step to reach the above goal, we acquired online the DXF of a wood clock "Genesis" by Clayton Boyle [12]. The clock was designed for hobbyists with the components cut manually with a scroll saw or a router. High-quality plywood was recommended for making the main components such as the gears. This is an ideal case to demonstrate the gear and clock making capability of waterjet in terms of fast turnaround and precision. The DXF files of the Genesis components were imported to LAYOUT and compiled in MAKE. 


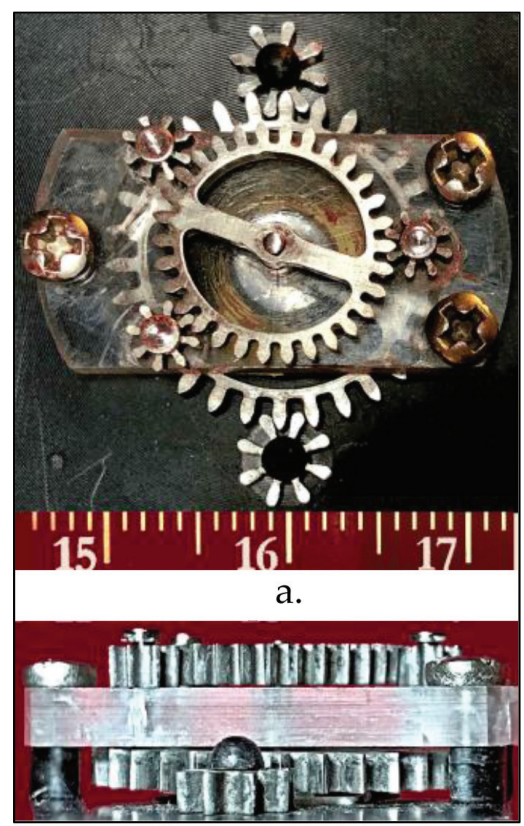

b.

Figure 11. Cycloidal gear set (a) Top view (b) Side view.

All the components of the Genesis clock were then cut on a MAXIEM waterjet system in the OMAX Demo Lab in just hours as opposed to days using the scroll saw. Figure 12 illustrates the assembled wood clock. The faces of the hour (lower left), minute (middle), and second (right) gears were cut from a thin stainless steel sheet. The clock is controlled by the adjustable length of the pendulum. The clock is driven by a $3.2 \mathrm{~kg}$ stainless steel bar that turns a click wheel attached to the back of the minute gear via a fish line. A small aluminum bar serves as the counter balance to straighten the fish line as the clock runs. Refer to Reference 12 for a detailed description of the clock.

For large bevels and countersinks, the A-Jet with a range of tilt angles from 0 to $60^{\circ}$ to the vertical can be used. Figure 13 illustrates a pair of beveled titanium honeycomb parts with 65 and $45^{\circ}$ edge bevel angles, respectively; both the facesheet and the core were made of titanium. Note that cutting titanium honeycomb presents a considerable challenge to most machine tools. CNC hard tools tend to deform the thin core material whereas lasers and EDM must cut slowly to minimize the HAZ. 


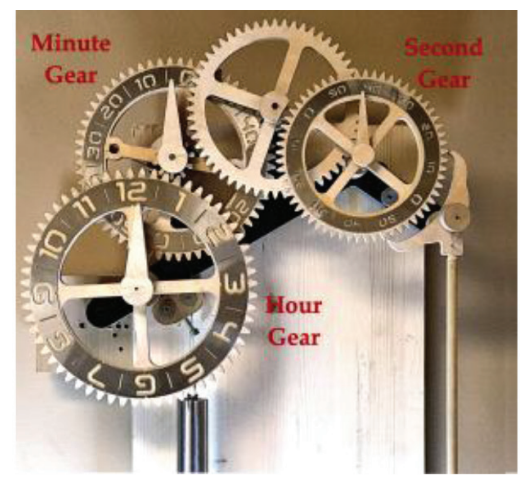

a.

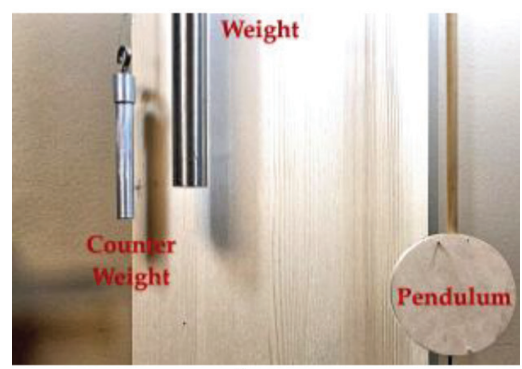

b.

Figure 12. "Genesis" wood clock [12] (a) Top half (b) Lower half.

By combining the operations of the Rotary Axis and the A-Jet, complex 3D parts can be readily machined. One of the useful applications is to machine "fish mouth" weld joints for metal pipes, large and small, as illustrated in Figure 14. The joints are often cut with plasma cutting machines that leave a large HAZ on the cut edges. Removal of the HAZ often is done manually, leading to high labor costs and slow turnaround. The Intelli-MAX Software Suite has built-in programs to prepare tool paths for weld joints that can be cut with one of the JMCs. The as-cut joints are weld ready without the need of any secondary processing.

Another application is to machine inclined holes such as those used in aircraft engines [3]. For modern aircraft engines operating at very high temperature, there is a need for drilling inclined and shaped air breathing holes to achieve maximum cooling. The current practice requires a two-step process to drill inclined and shaped holes on TBC coated metal. First, the nonconductive TBC is removed with a laser and the hole in the substrate is drilled with an 


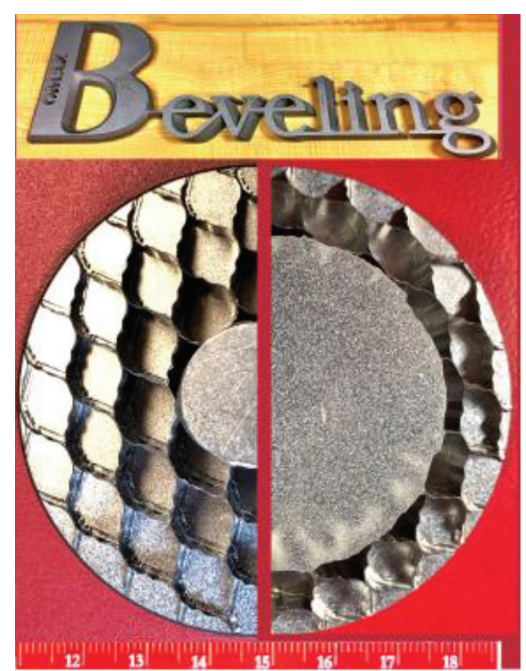

a.

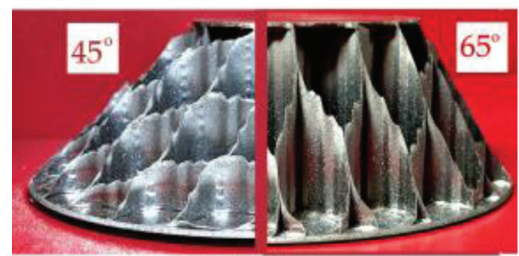

b.

Figure 13. Beveled titanium honeycomb parts (a) Top view (b) Side view.

EDM process. The EDM process is very slow in order to minimize the HAZ damage. The AWJ was applied successfully to drill such holes on refractory metals with and without thermal barrier coating, as illustrated in Figure 15. In the absence of the HAZ, the AWJ drills holes much faster than $\mathrm{CNC}$ tools. By mounting the workpiece on the Rotary Axis, any inclined angle of holes can be drilled. The geometries of the holes were drilled by controlling the tilting of the A-Jet. Within certain limitations, the inclined angle and the shape can vary simultaneously along the hole axis. The AWJ nozzle consisted of a 0.18-mm ID diamond orifice and a 0.38-mm ID mixing tube. Garnet of 220 mesh with a flow rate of $45 \mathrm{gr} / \mathrm{min}$ was used. Seven hole geometries were drilled with a single nozzle on these samples to demonstrate the versatility of the AWJ in hole drilling. Most important, there was no delamination between the coatings and substrates and no HAZ on the hole edges on the substrates. 


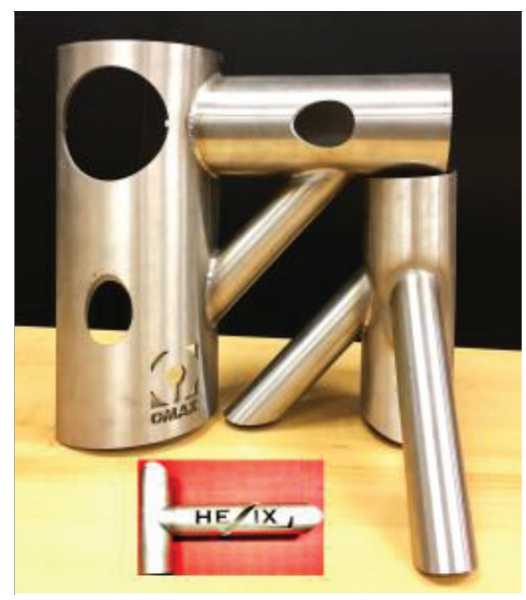

Figure 14. AWJ-cut "Fish mouth" weld joints.

\subsubsection{Milling of glass mirrors}

One of the more interesting applications for abrasive waterjets is controlled depth milling. Instead of cutting through the workpiece, the abrasive waterjet is traversed at a high speed across the part's surface. This causes the jet's kerf to change from a through cutting cross section to a grooving and then to an etching cross section. As the relative traverse rates increase between the nozzle and workpiece's surface, the penetration depth decreases. Precise depth control is achieved through a multi-pass process when, like with traditional milling operations, the final depth is achieved by walking the milled surface down to the final target depth. This is achieved by choosing a process where the amount of material removed per milling pass is less than the target depth tolerance. Depth control on the order of $0.03 \mathrm{~mm}$ can be achieved with the correct combination of process parameters.

When milling glass materials, the goal is to diffuse or reduce the power being applied to the surface of the part from a glass fracturing risk perspective. It is well known that cutting glass without abrasives results in fracturing the glass. Milling is no different, except that the fracturing tends to have more of a spalling damage. The key is choosing a set of process parameters where if the abrasive feed was interrupted, then glass will not break. This is achieved by using higher standoff distances on the order of 150-300 $\mathrm{mm}$, orifice diameters less than $0.2 \mathrm{~mm}$, mixing tube diameter to orifice diameter ratio's on the order of 10:1, and mixing tube lengths $100-300 \mathrm{~mm}$, with jet pressures in the 70-200 MPa. The abrasive mass flow rate to waterjet mass flow rate ratio ranges from $25-100 \%$. One of the keys is the traverse rates from $0.02 \mathrm{~m} / \mathrm{s}$ to over $8 \mathrm{~m} / \mathrm{s}$. The higher the traverse rate, the more precise the depth control. The higher traverse rates are easier to achieve by spinning the work piece on a turntable. 


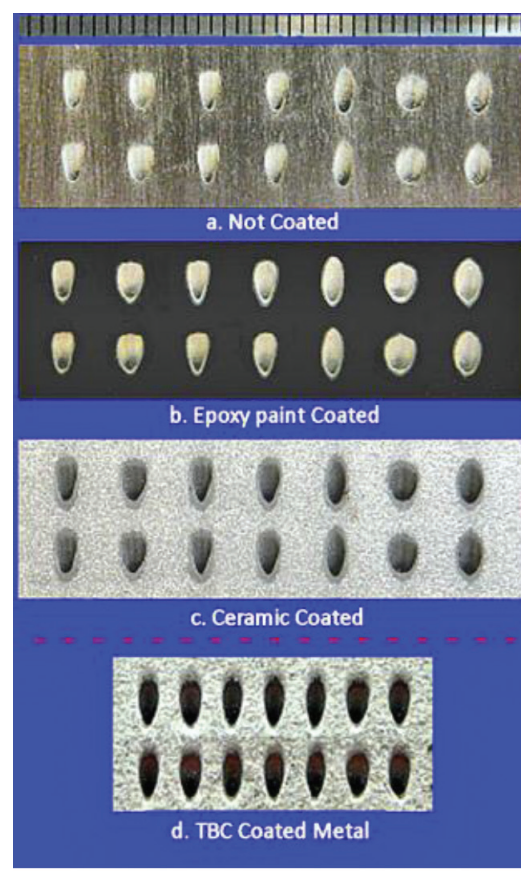

Figure 15. Inclined shaped holes on refractory metals.

One of the applications that the abrasive waterjet milling process has been successfully applied to is reducing weight in glass materials for ultralight-weight mirrors [13]. Samples include a $250 \mathrm{~mm}$ (major axis) elliptical mirror with pockets milled to a depth of $9.5 \mathrm{~mm}$ (Figure 16) and a $305 \mathrm{~mm}$ wide mirror made from $5.3 \mathrm{~mm}$ thick Ultra Low Expansion (ULE) glass with pockets milled to a depth of $3.6 \mathrm{~mm}$ (Figure 17). This mirror design was for testing of the active bending concept to change its focal point for phasing together multiple mirrors together for the James Webb telescope program.

These mirrors were milled with a milling process where the relative traverse rates were about $8 \mathrm{~m} / \mathrm{s}$. At these speeds, slowing the jet down to change directions without causing the jet to mill deeper as the jet speed decreased is mechanically impossible to accomplish. To solve this problem a mask with the lightweighting Isogrid pattern was placed on top of the glass, and the abrasive waterjet milling process is rastered across the entire surface of the mask. The mask was made from steel, and the relative erosion rate between the glass and steel is about 40 to 1 . This allows for the mask to be reused on multiple parts before needing to be replaced. 
01

02

03

04

05

06

07

08

09

Figure 17. $305 \mathrm{~mm}$ wide mirror made from $5.3 \mathrm{~mm}$ thick ultra-low expansion glass with pockets milled to $3.6 \mathrm{~mm}$ deep.

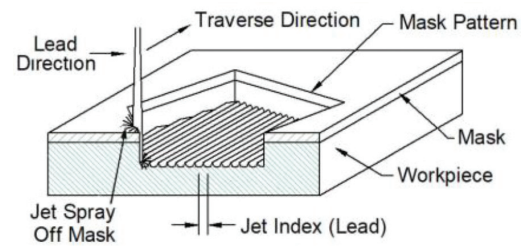

Figure 18 shows an artistic milled pocket pattern that can be easily replicated dozens of times using the same milling mask.

Figure 19 illustrates how the jet rasters across the mask's surface. The glass is milled where all of the openings in the masks are located. Very intricate patterns can be milled into the glass surface. As a side note, one of the other advantages the abrasive waterjet machine has, is that the very same tool used to mill the glass can be used to cut the mask pattern. After each pass of the abrasive waterjet, the centerline of the jet is laterally indexed, as shown in Figure 20. When the index distance is approximately $70 \%$ of the mixing tube diameter, the milled surface produced is smooth and flat.

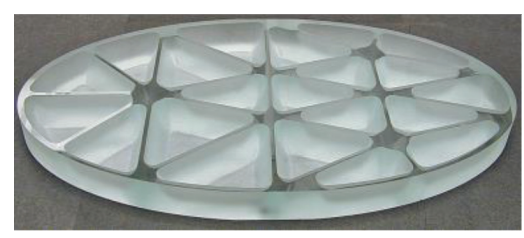

Figure 16. $250 \mathrm{~mm}$ (major axis) elliptical mirror with pockets milled to $9.5 \mathrm{~mm}$ deep.

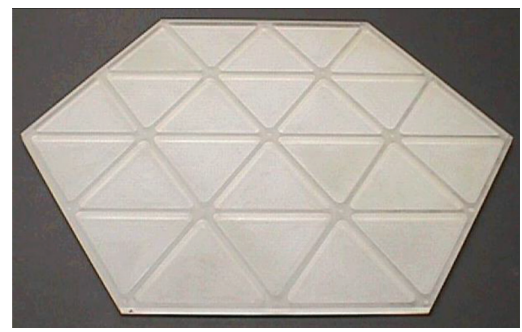


Figure 19. Superimposing successive milling passes to generate flat surfaces.

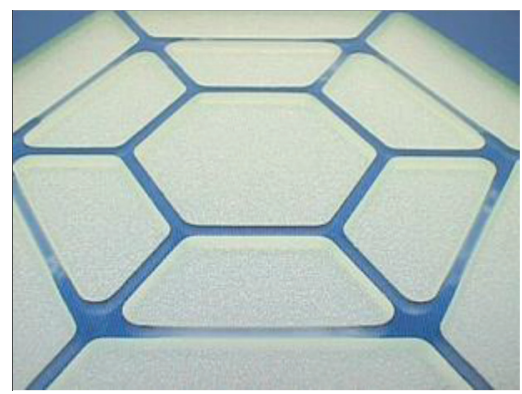
examples by assembling multiple layers of AWJ-machined pieces.

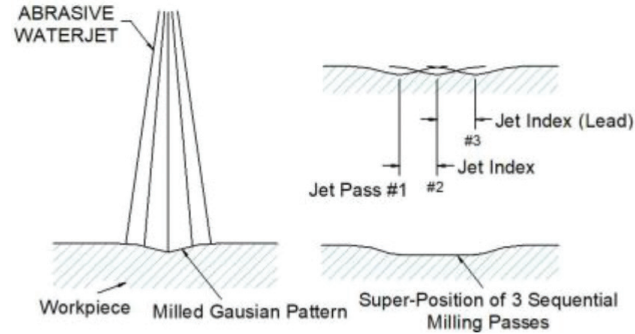

\footnotetext{
Figure 19. Superimposing successive milling passes to generate flat surfaces.
}

Glass that is often used as a comparison material for industrial comparative testing is a great material to demonstrate the versatility of AWJ. Known as a strong and brittle material, glass has a variety of applications across industries, including the creative sector. Exploration to generate artworks that investigate the waterjet process in the medium of glass was conducted. Working in a variety of scales the process remains the same with slight considerations regarding the delicacy, intricacy and complexity of the design [14]. Figure 21 illustrates two artwork

The design process can start in a variety of different ways, such as importing a vector file from any software capable of saving a drawing as a vector file (e.g., Rhino, AutoCAD, Illustrator and SolidWorks). The process of cutting is a two dimensional process and therefore requires a single outline. The initial programming is undertaken in various softwares and nested into the machine's software before cutting. The files are made and saved as a vector such as a DWG or DXF file. In work such as the "Scrutiny" handwriting was photographed and saved as a 


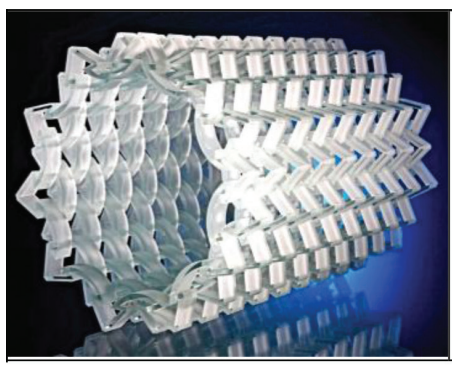

a

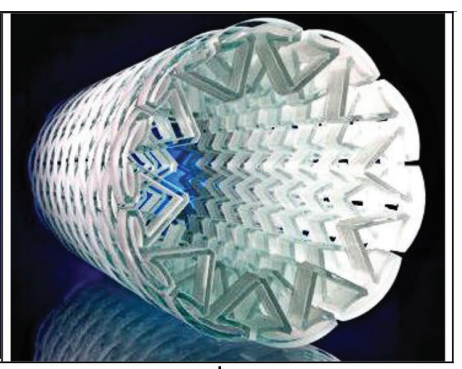

b

Figure 21. AWJ-machined artwork examples (a) Multi-layers of AWJ-cut glass (b) "Intertwine" glass sculpture.

JPEG and imported into the OMAX Intelli-TRACE software, where the writing was adapted within the software to fit within a given surface area [15].

The process is able to capture detail and work to tight tolerances and variable angles to effectively capture the handwriting. The AWJ can cut through stacks, working with glass thicknesses from $1 \mathrm{~mm}$ to over $65 \mathrm{~mm}$ using a variety of soda-lime, clear float glass and various artist glass stacks such as Bulleye. ${ }^{4}$

A variation in speed of abrasive flow, standoff distance, and how the machine is set up along with the order and direction of cutting can have effect on obtaining a successful outcome. Optimum pump pressures depending on the work undertaken varies between 11,000 and 58,000 psi. Higher pressure pumps have been used but with the application of multiple pierce points and variation of pressure from high to low, a lower pressure pump has proved more suitable due having to ramp from low to high pressure multiple times. Maintaining a consistency of pressure and abrasive is crucial in cutting glass. In cutting the handwriting, rhino board was used for the more delicate forms with water not covering the head in case a splash fractured the glass. Other handwriting at $2 \mathrm{~mm}$ thickness and not as complex, the work was cut underwater. Figure 22a-c illustrates the processes for machining handwriting on glass. ${ }^{5}$

For 5-axis cutting, the consideration with glass is how the material is held in place, as well as the order and priority of cutting. Most work is cut sitting on a surface tilled to reduce the residual wastes falling away. Another consideration is "taper lock," which can trap the form within the waste material. There is a lot more risk with a brittle material such as glass; residual stress within the material can causing internal fracturing especially in thicker glass material. Cutting a form in glass can have different programming to that of a metal form and its set up is crucial to a successful outcome.

${ }^{4} \mathrm{~A}$ brand of fusing glass that allows various colored glass to be used together that have the same coefficient that make the glass compatible with each other.

5Photography credit: Simon Bruntnell 


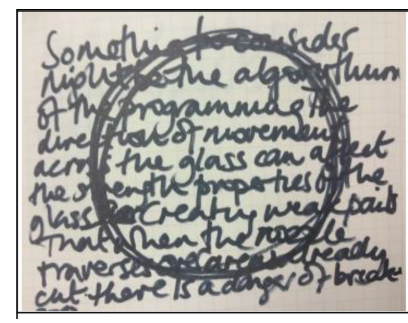

a.

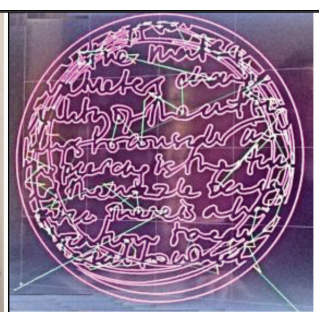

b.

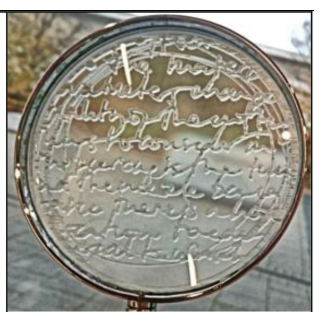

C.

Figure 22. Processes for AWJ-machining of handwriting on glass [15] (a) Initial drawing sketch (b) Tool paths and (c) Micro glass handwriting.

\subsubsection{Piercing of composites}

Composites, laminates, and brittle materials have long been difficult materials to process by conventional machine tools such as mills and lathes as well as abrasive water jets and other beam cutting technologies. Most of the issues involved in shaping involve either peculiarities with the materials' heat sensitivity, brittleness, low tensile strength or its abrasive nature. Rapid wearing of alloy drills has been one of the main concerns that degraded the precision and repeatability of machined features. Early tests revealed similar damage took place during the initial hole piercing process. Considerable efforts were subsequently made in an attempt to understand and mitigate such damage [3, 16-17]. It was discovered that damage occurred whenever the buildup of stagnating pressure inside blind holes exceeds the tensile/adhesive strength of composites/ laminates. Based on the above understanding, novel processes to minimize the stagnating pressure were developed for piercing composites/ laminates without inducing damage. The Turbo (patented) and Mini Piercers were developed for AWJ drilling of large and small holes, respectively. Figure 23 illustrates AWJ-machined internal features that require piercing on composite (G10), laminate (aluminum), brittle materials (glass and silicon wafer) with no damage.

Another advantage of using AWJ to machine composites is that the nozzle, unlike drill bits, does not come in direct contact with the workpiece. In other words, the nozzle wear is independent of the property of composite workpiece. For certain composites that are highly abrasive, excessive and rapid wear was experienced by the drill bits. Such rapid wearing of the drill bits tends to degrade the precision and repeatability of the machined features [18]. On the other hand, the AWJ nozzle wears considerably slower than the drill bits do. For extremely precise parts, AWJ can be readily used as a near-net shaping tool. The part can then be finished by light trimming with a precision hard tool. As such, the tool life can be greatly extended.

\subsubsection{Patient-specific orthopedics and prosthetics}

At present, most orthopedic and prosthetic implants are mass produced with limited sizes to achieve an average fit for individual patients. Since the implants are not tailored to the specific patient, it is not possible to optimize the implant operation for an optimum match. Recently, 


\section{Figure 23. Piercing with Turbo and mini piercers.}

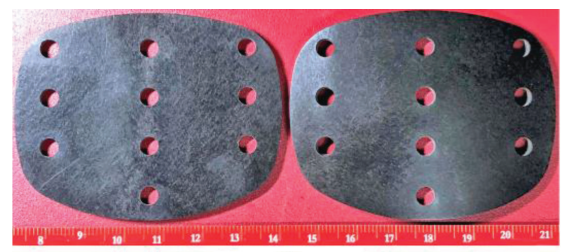

a.

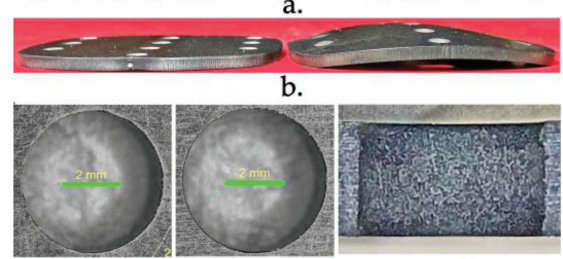

c.

Figure 24. AWJ-machined cranial implants made from PEEK with fiber reinforcement (a) Top view (b) Side view and (c) Top, bottom, and side view.

there has been strong advocacy for manufacturing patient specific implants for optimum fit, with a slogan of "one patent, one implant." Waterjet technology with its technological and manufacturing merits is most suitable for manufacturing such implants cost effectively Waterjets are expected to lower the manufacturing cost of implants because of its no tooling requirement. As a cold cutting tool, all parts can be machined including secondary processes,

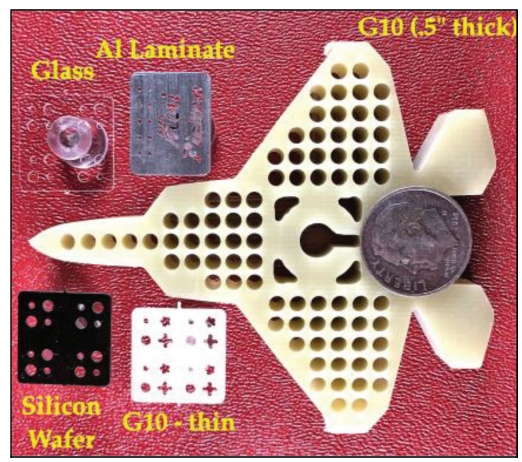

b. 
if needed, in a matter of minutes or hours, depending on the complexity of the parts. Such fast turnaround is a must for in-situ implant operations. Furthermore, a mobile waterjet system has been applied successfully in remote areas such as the battlefield for rapid response repair [19]. The ruggedness of the system would facilitate setting up waterjet systems in remote areas for machining implants to broaden the reach of quality healthcare to underprivileged populations.

The applications of AWJ machining of biomedical components made of biocompatible metals such as titanium and stainless steel have been given elsewhere [11, 20]. An example of an AWJ-machined titanium mesh cage is illustrated in Figure 8. A relatively new biocompatible material, Poly-Ether-Ether-Keytone (PEEK), has been shown to be a superior replacement of titanium implants in terms of avoidance of superior biocompatibility, allergic tissue reaction, and transparency to X-rays [21]. Success in applying waterjet for machining PEEK implants would greatly reduce the manufacturing costs together with fast turnaround. Figure 24 illustrates AWJ-machined internal features that require piercing on the PEEK material with carbon fiber reinforcement. On the right of Figure 24a and $\mathbf{b}$, the curved implant was thermally shaped at $316^{\circ} \mathrm{C}$. Figure $24 \mathrm{c}$ shows the micrographs of the top, bottom and side views of one of the holes. Note that the hole edges were cut cleanly with no fiber hanging out loosely.

\section{Conclusion}

With the commercialization of micro abrasive waterjet or $\mu \mathrm{AWJ}$ technology, the full capability has established for precision multimode machining of most materials from macro to micro scales for a wide range of part size and thickness. This "7 M" advantage of waterjet technology, together with cost effectiveness and fast turnaround, has greatly broadened manufacturing applications from R\&D, prototyping, to 24-7 production of both small and large lots. The technological and manufacturing merits of waterjet technology have elevated it as one of most versatile machine tools unmatched by others. Specifically, the material independence and low side force exertion on workpieces are two most outstanding technological merits. A collection of AWJ-machined samples, made from a wide range of materials from metal, nonmetal, and anything in between, were presented to demonstrate the versatility of waterjet technology for a broad range of applications. In particular, machining many such examples presents considerable challenge to other machine tool in terms of material property, part geometry, tool performance, equipment/production costs, and machining/turnaround time.

It is concluded that recent advancement has elevated waterjet as a mainstream machine tool, often competing with lasers EDM, and others on equal footings. For certain applications, waterjet out performs its competitors. For cutting heat sensitive materials with low tolerance in heat damage, waterjet is at least 10 times faster than lasers and EDM.

\section{Acknowledgements}

This work was supported by an OMAX IR\&D fund and NSF SBIR Phase I and II Grants \#1058278. Any opinions, findings, and conclusions or recommendations expressed in this 
material are those of the authors and do not necessarily reflect the NSF's views. The authors wish to thank the technicians in the Demonstration Laboratory for assistance in machining some of the parts illustrated in this article.

\section{Author details}

(Peter) H.-T. Liu' ${ }^{1 *}$, V. Cutler ${ }^{2}$, C. Raghavan ${ }^{1}$, P. Miles ${ }^{1}$, E. Schubert ${ }^{1}$ and N. Webers ${ }^{1}$

*Address all correspondence to: peter.liu@omax.com

1 OMAX Corporation, Kent, Washington, United States

2 Independent Waterjet Specialist and Glass Artist, Hampshire, United Kingdom

\section{References}

[1] Liu H-T, Schubert E. Micro abrasive-waterjet technology. In: Kahrizi M, editor. Micromachining Techniques for Fabrication of Micro and Nano Structures. INTECH Open Access Publisher; 2012. pp. 205-234. DOI: 10.5772/1364

[2] Liu PH-T. Mint: Precision machining of advanced materials with abrasive waterjets. IOP Conf. Series: Materials Science and Engineering, 164, 2017. 012008 DOI:10.1088/1757899X/164/1/012008 (5th Global Conference on materials Science and Engineering, 8-11 November 2016, Tunghai University, Taiwan)

[3] Liu H-T. "7M" advantage of abrasive waterjet for machining advanced materials. Mint: Journal of Manufacturing and Materials Processing. 2017; 1(11): 1-19 MDPI, Basel, Switzerland. DOI: 10.3390/jmmp1010011. http://www.mdpi.com/2504-4494/1/1/11/pdf

[4] (Peter) Liu H-T. (2015) Abrasive-waterjet machining of most materials from macro to micro scales, In: Proceedings 2015 TechConnect World Innovation Conference and Expo; 14-17 June 2015; Washington DC. pp. 39-42

[5] Liu H-T. Versatility of micro abrasive waterjet technology for machining nanomaterials. Dekker Encyclopedia of Nanoscience and Nanotechnology, 3rd ed. 2017. pp. 1-18. DOI: 10.1081/E-ENN3-120054064

[6] Watanoa S, Imada Y, Hamadab K, Wakamatsub Y, Tanabeb Y, Davec RN, Pfefferc R. Mint: Microgranulation of fine powders by a novel rotating fluidized bed granulator. Mint: Powder Technology. 2003;131:250-255

[7] Liu PH-T. Roles of abrasives in AWJ meso-micro machining. In: Proceedings of 22nd International Conference on Water Jetting; 3-5 September 2014; the Netherland. pp. 151-165

[8] Liu PH-T. Roles of taper compensation in AWJ precision machining. In: Proceedings of the. 23rd International Conference on Water Jetting; 16-18 November 2016. Washington: Seattle. pp. 33-46 
[9] Kluger JM, Slocum AH, Sapsis TP. Beam-based Nonlinear US Patent No. 9599180 B2; 2016

[10] Kluger JM, Sapsis TP, Slocum AH. High-resolution and large force-range load cell by means of nonlinear cantilever beams. Precision Engineering, 43, 2016. 241-256 (http:// dx.doi.org/10.1016/j.precisioneng.2015.08.003)

[11] Liu H-T, Olsen JH. Application of AWJ for 3D machining. In: Proceedings of the 2013 WJTA-ICMA Conference and Expo; 9-11 September 2013; Houston, Texas

[12] Boyle C. Genesis [Internet] 2018. Available from: http://lisaboyer.com/Claytonsite/ Genesispage1.htm [Accessed: January 07, 2018]

[13] Miles P. Lightweighting large optics with abrasive Waterjets. In: Proceedings of SPIE, Novel Optical Systems and Large Aperture Imaging, 3430; 1998

[14] Cutler V. New Technologies in Glass. London: Bloomsbury; 2012. 128 p. ISBN 978-14081395-4-7

[15] Webers N, Cutler V. Interpreting handwritten scrawl. In: Proceedings of 23rd International Conference on Water Jetting; 16-18 November 2016; Seattle, Washington. pp. 99-109

[16] Liu H-T, Schubert E. Piercing in delicate materials with abrasive-waterjets. Mint: The International Journal of Advanced Manufacturing Technology. 2009;42(3-4):263-279. DOI: 10.1007/s00170-008-1583-5

[17] Liu H-T, Schubert E, McNiel D, Soo, K. Applications of abrasive-waterjets for precision machining of composites. In: Proceedings of SAMPE 2010 Conference and Exhibition, 17-20 May 2010; Seattle, Washington; 2010

[18] Khashaba UA. Drilling of polymer matrix composites: A review. "Mint" Journal of Composite Materials. 2013;47(15):1817-1832. DOI: 10.1177/0021998312451609

[19] Anderson M, editor. Watercutting in Wartime [Internet]. 2013. Available from: http:// sme.org/MEMagazine/Article.aspx [Accessed: Decemeber 29, 2017]

[20] Liu H-T. Abrasive-waterjet technology for biomedical applications, In: Proceedings 21st Internal Conference on Water Jetting; 19-21 September 2012; Ottawa, Canada. pp. 99-112

[21] Kurt SM, Devine JN. PEEK biomaterials in trauma, orthopedic, and spinal implants. Mint: Biomaterials. 2007;28(32):4845-4869 
